\title{
'Patient reassurance is paramount'
}

\author{
By Kate Quinlan
}

\section{Are dental practitioners confident to approach and manage}

patients with dementia, taking into account the new guidance published by the FGDP(UK)?

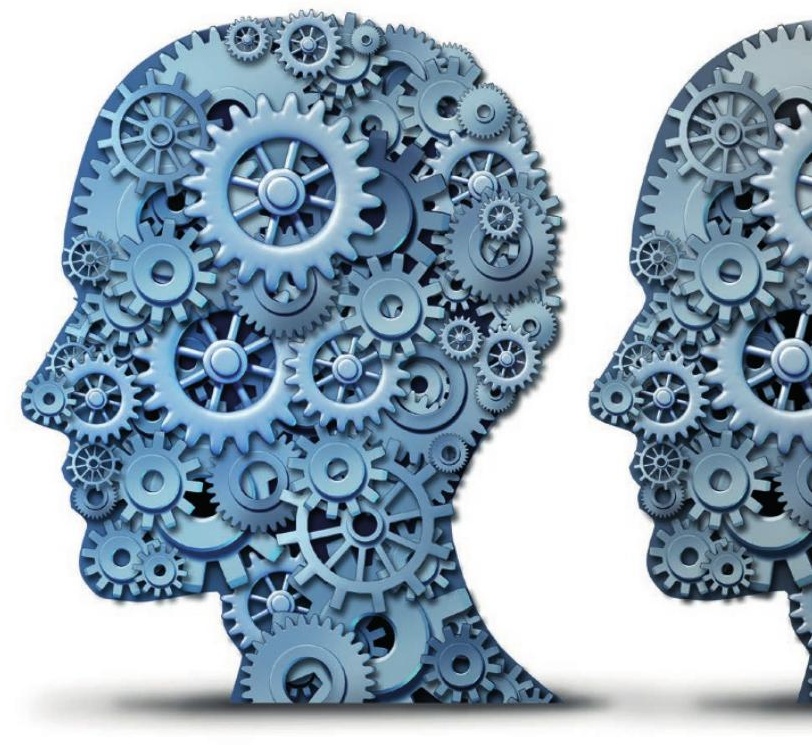

I n October the Faculty of General Dental Practice UK (FGDP[UK]) published new guidance on the practice of dentistry for patients with dementia (available via www.fgdp.org.uk).

With around 850,000 people in the UK with the condition $-5 \%$ of the population - the Faculty says that dental professionals need to understand it, and adapt their patient management and clinical decisions accordingly. The guidelines cover:

- The epidemiology and diagnosis of dementia, and its implications for dental professionals

- Principles of care management, including patient identification, competence and referrals, communication, consent and capacity

- Clinical care, including history taking, treatment planning, care delivery and prescribing

- Site-specific considerations for dental practices, care homes and domiciliary care.

In this feature, three dentists describe how they approach patients with dementia; the challenges they are faced with when treating patients with dementia; and their response to the FGDP(UK)'s guidelines. Also in this issue's Upfront is an account written by Glenys Bridges on dementia-friendly dental practice from the patients' perspective, based on personal experience.

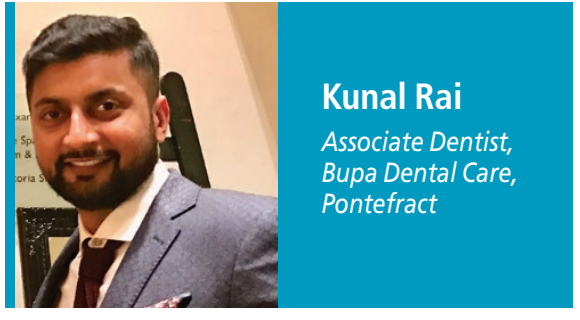

How do you approach patients with dementia?

- First we work out the stage of dementia - so that we can be prepared for any aggression, confusion or any behavioural changes

- Check if the patient has a carer - if so, involve the carer in the treatment plan

- Patient consent - be aware of patients who attend on their own without a carer. Does anybody have a power of attorney or authority to make decisions on the patient's behalf?

- Avoid any dental jargon. Keep the language simple and to the point

- Give the patient all information in writing, which is easy to understand (layman's terms) so they consent freely (informed consent)

- Make the carer aware of any signs and symptoms that indicate dental pain, such as disturbed sleep or problems with mastication

- Always respect them and treat them kindly with empathy

- Above all, we like to stay welcoming, be caring and friendly.
What are the challenges you face when treating patients with dementia?

- Patient aggression, irritation, medical history not filled in completely (leading to potential list of medication being missed out)

- Ensuring the patient completely understands the treatment plan - and consents

- Patient may not be able to communicate or show signs of any dental issues

- As there is reduced ability of concentration - patient may close their mouth during treatment, leading to potential injury or difficult examination

- The sound of a dental handpiece may cause the patient to become irritated and aggressive.

Would you be comfortable raising concerns about suspected dementia with a patient? (see section 3.2 in the guidelines, How to raise concerns and approach the subject of dementia). Yes, as we need to work in the patient's best interest. Obviously the first line of action would be to acquire the patient's consent and make them aware as to why this is necessary. Patient reassurance is paramount - showing empathy helps the patient to feel relaxed.

Do you feel dental professionals are well placed to pick up on suspected dementia? Yes, dental professionals are able to pick up suspected dementia due to closely working with patients on a daily basis. These include noting down early signs, especially in cases where a patient has been at a practice for a long time.
9

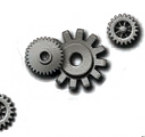

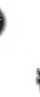

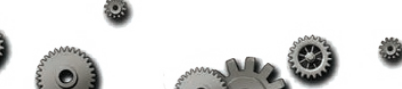




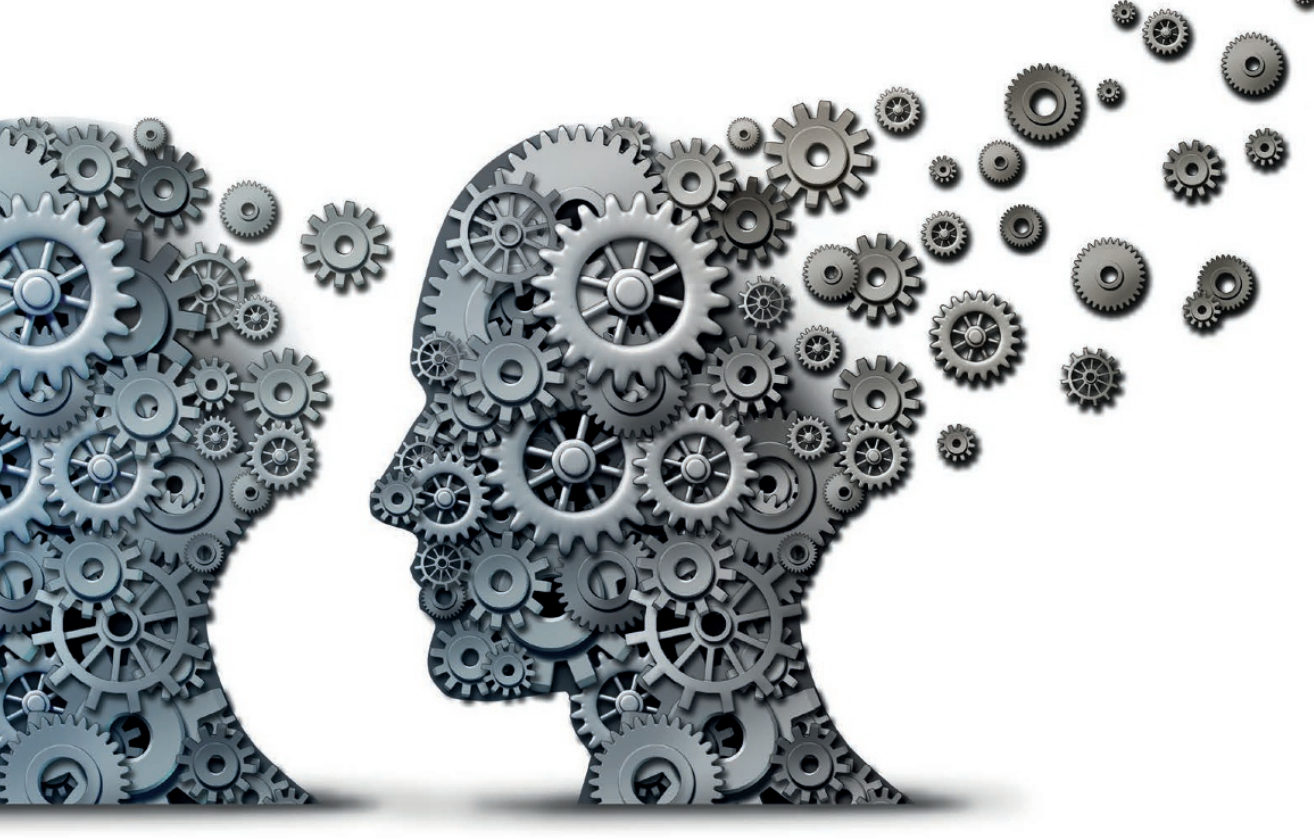

patient co-operation is the key in managing patients living with dementia. Understanding the individual patient, and what works for them, is the key to successful treatment. This includes the duration of the appointment, the timing (morning or afternoon), communication, dependence on carers, tolerance for different types of treatment, ability to cope with the dental environment (such as noisy and crowded waiting rooms) and access issues.

What are the challenges you face when treating patients with dementia? One of the biggest challenges treating new patients with dementia (for example, a patient living in a nursing home who you haven't previously

Have you used the VERA communication technique? (Validation/Emotional context/ Reassurance/Activity) Yes. This technique in general is used to an extent and subconsciously when communicating with the majority of my patients, especially Validation, Emotional and Reassurance.

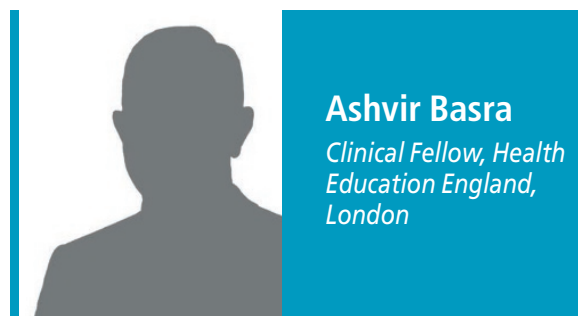

How do you approach patients with dementia? I work in the special care dental services, so feel well placed to see and manage dementia patients. I will always try to see a patient in the dental chair and use the initial appointment to gauge their cooperation, level of understanding, capacity to consent as well as determine how much care they are able to perform independently. I will try to explain everything well during their dental visits, and fully understand the social history of the patient (ie care home resident or family care involvement).

What are the challenges you face when treating patients with dementia?

- The capacity to consent can be an issue and determining whether best interest pathways are required

- Trying to explain procedures to patients with dementia - it is hard to know how much is understood and retained

- Actual patient management can be hard - to keep a patient in an appropriate position in order to carry out an examination or simple treatment.

Would you be comfortable raising concerns about suspected dementia with a patient? (see section 3.2 in the guidelines, How to raise concerns and approach the subject of dementia). I would be comfortable to ask probing questions about indicators of early dementia and broaching the topic of communicating with their GP, with their consent to do so.

Do you feel dental professionals are well placed to pick up on suspected dementia? Dental professionals could be well placed to pick up on suspected dementia and signpost appropriately; however, I feel that the current climate and perverse incentives in general dental practice make this a difficult request for dental colleagues in general practice. Commissioning support as an incentive could work and indeed is being piloted in England.

Have you used the VERA communication technique? (Validation/Emotional context/ Reassurance/Activity) I have used this technique without realising it as a recognised communication technique. This is just something I use from previous experience.

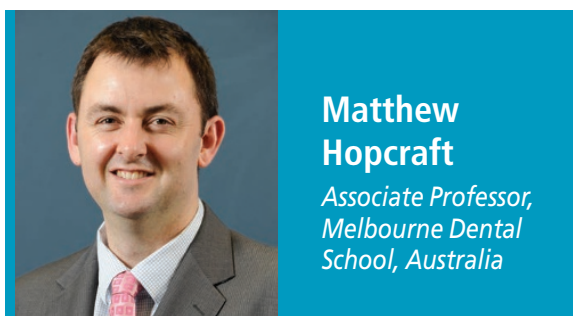

How do you approach patients with dementia? It's important to recognise that seen) is helping them to understand what we are doing. Patients living with dementia often have difficulty with new environments and people, so a dental visit with a new dentist in a nursing home presents a huge challenge. The other really significant issue is obtaining informed consent. This can be difficult when there has not yet been a diagnosis of dementia, but the patient is displaying symptoms.

Would you be comfortable raising concerns about suspected dementia with a patient? (see section 3.2 in the guidelines, How to raise concerns and approach the subject of dementia). This is difficult, because it is often hard to distinguish the early signs of dementia from normal ageing, and no-one wants to make a mistake and possibly embarrass the patient. However, with more experience, it becomes easier to recognise dementia and raise this with patients and/or family members.

Do you feel dental professionals are well placed to pick up on suspected dementia? I don't think that dental professionals are well trained to recognise suspected dementia, or have the necessary referral pathways to assist with diagnosis. Dental education is lagging broadly in the area of gerodontics and special care dentistry, and specifically in the management of patients living with dementia. There is also a lack of continuing professional development that focuses on this important (and growing) patient group.

If you would like to contribute to our next Perspectives feature, please send your details to k.quinlan@nature.com. 